\title{
Synchronization of the Fractional-Order Brushless DC Motors Chaotic System
}

\author{
Shiyun Shen ${ }^{1}$ and Ping Zhou ${ }^{1,2}$ \\ ${ }^{1}$ Center of System Theory and Its Applications, Chongqing University of Posts and Telecommunications, \\ Chongqing 400065, China \\ ${ }^{2}$ Key Laboratory of Network Control and Intelligent Instrument of Ministry of Education, \\ Chongqing University of Posts and Telecommunications, Chongqing 400065, China
}

Correspondence should be addressed to Ping Zhou; zhouping@cqupt.edu.cn

Received 3 April 2016; Accepted 20 July 2016

Academic Editor: M. Junaid Khan

Copyright (C) 2016 S. Shen and P. Zhou. This is an open access article distributed under the Creative Commons Attribution License, which permits unrestricted use, distribution, and reproduction in any medium, provided the original work is properly cited.

Based on the extension of Lyapunov direct method for nonlinear fractional-order systems, chaos synchronization for the fractionalorder Brushless DC motors (BLDCM) is discussed. A chaos synchronization scheme is suggested. By means of Lyapunov candidate function, the theoretical proof of chaos synchronization is addressed. The numerical results show that the chaos synchronization scheme is valid.

\section{Introduction}

The brushless direct-current motors (BLDCM) have many advantages over brushed direct-current motors [1-7], including more torque per watt, high torque per weight, longer lifetime, lower noise, lower electromagnetic interference, and high reliability. So, the BLDCM has been used diffusely in industrial automation and manufacturing engineering, for example, computer hard drives and CD/DVD players, electric vehicles and hybrid vehicles, motion control, positioning or actuation systems, and micro radio-controlled airplane. Recently, chaotic behavior in the brushless directcurrent motors (BLDCM) has been reported [1-7], and many researchers investigated chaos synchronization and chaos control for the BLDCM chaotic system [1-7]. Chaos is useful in many applications, for example, in image steganography $[8,9]$, authenticated encryption [10], and chaotic communications [11]. It must be pointed out that chaos synchronization and chaos control are usually a prerequisite in chaos application.

On the other hand, based on the integer-order BLDCM chaotic system reported by Ge et al., a fractional-order BLDCM chaotic system [12] has been proposed by Zhou et al.
By the adaptive control, back stepping design, and Lyapunov stability theory, the authors $[2,3]$ proposed some schemes of chaos synchronization and chaos control for the integerorder BLDCM chaotic system. Based on the generalized Gronwall inequality, Zhou et al. [12] presented two chaos control strategies for the fractional-order BLDCM chaotic system. To the best of our knowledge, there are seldom results on chaos synchronization for the fractional-order BLDCM chaotic system. Motivated by the above discussions, we investigate chaos synchronization for the fractional-order BLDCM chaotic system in this paper. Based on the extension of Lyapunov direct method for nonlinear fractional-order system $[13,14]$, a chaos synchronization scheme is proposed. By a Lyapunov candidate function, the theoretical proof of chaos synchronization is provided. Simulation results demonstrate the effectiveness of the synchronization scheme in our paper.

The rest of this paper is as follows: Section 2 introduces the fractional-order BLDCM chaotic system, and chaotic attractors are given. Chaos synchronization for the fractionalorder BLDCM chaotic system is discussed in Section 3, and simulation results are obtained. Finally, Section 4 concludes the work. 

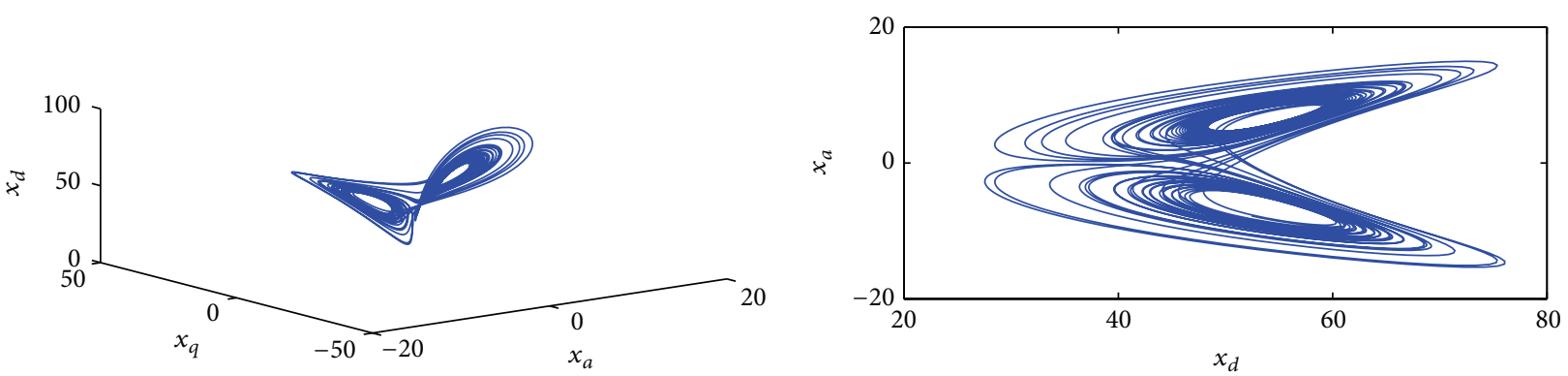

FIGURE 1: A chaotic attractor in the fractional-order BLDCM system (1) for $q=0.967$.
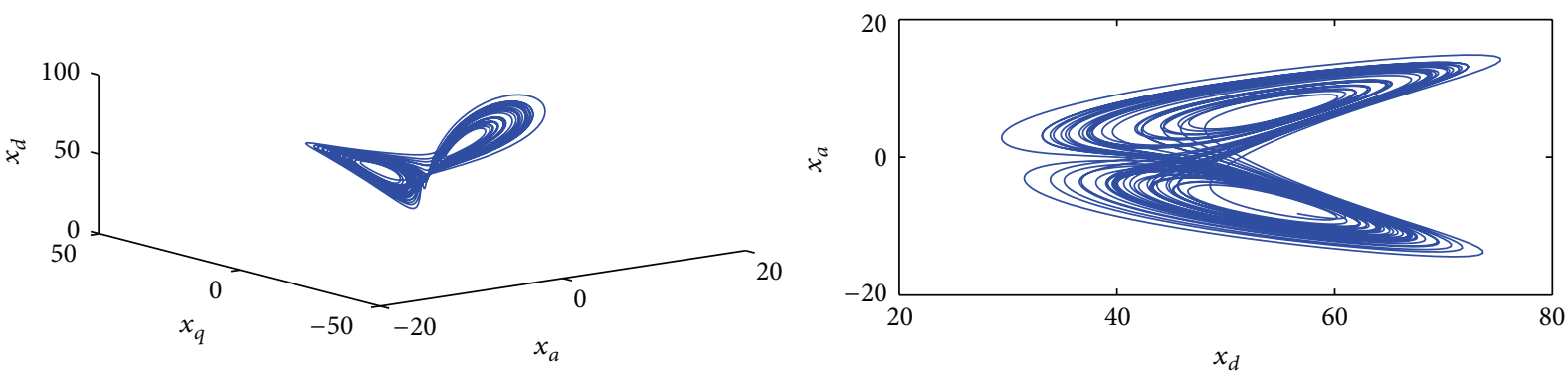

FIGURE 2: A chaotic attractor in the FO-BLDCM system (1) for $q=0.975$.

\section{The Fractional-Order BLDCM Chaotic System}

Recently, a fractional-order BLDCM chaotic system was reported by Zhou et al. [12], and this chaotic system can be described as follows:

$$
\begin{aligned}
& { }_{0}^{C} D_{t}^{q} x_{d}=-\sigma x_{d}+x_{q} x_{a}, \\
& { }_{0}^{C} D_{t}^{q} x_{q}=-x_{q}+\beta x_{a}-x_{d} x_{a}, \\
& { }_{0}^{C} D_{t}^{q} x_{a}=\gamma\left(x_{q}-x_{a}\right),
\end{aligned}
$$

where $0.96<q \leq 1$ is the fractional order and ${ }_{0}^{C} D_{t}^{q} x_{i}=$ $\Gamma^{-1}(1-q) \int_{0}^{t}(t-\tau)^{-q} d x_{i}(\tau)(i=d, q, a) . x_{d}$ is the direct axis current of the motor, $x_{q}$ is quadrature axis current of the motor, and $x_{a}$ is the angular velocity of the motor. The motor parameters are chosen as $\sigma=0.875, \beta=55$, and $\gamma=4$. The authors obtained the maximum Lyapunov exponent on varying $q$ in [12], and system (1) exhibits chaotic behavior if $0.96<q \leq 1$. Now, we can choose $q=0.967$ and $q=$ 0.975 and obtain the maximum Lyapunov exponent as 0.7767 and 0.8954 , respectively. The positive maximum Lyapunov exponent implies that fractional-order BLDCM system (1) is chaotic under $q=0.967$ and $q=0.975$, and the chaotic attractors are shown as Figures 1 and 2, respectively.

\section{Synchronization for the Fractional-Order BLDCM Chaotic System}

In this section, chaos synchronization for the fractional-order BLDCM chaotic system (1) is considered. First, we recall some results for the Caputo derivative.
Lemma 1 (see [15]). For absolutely continuous functions $a(t)$ and $b(t)$, one can obtain the following equality:

$$
\begin{aligned}
{ }_{0}^{C} D_{t}^{q} & {[a(t) b(t)]-a(t){ }_{0}^{C} D_{t}^{q} b(t)-b(t){ }_{0}^{C} D_{t}^{q} a(t) } \\
= & \frac{-q}{\Gamma(1-q)} \\
\cdot & \int_{0}^{t} \frac{d \tau}{(t-\tau)^{1-q}}\left[\int_{0}^{\tau} \frac{d a(\omega)}{(t-\omega)^{1-q}} \int_{0}^{\tau} \frac{d b(\rho)}{(t-\rho)^{1-q}}\right],
\end{aligned}
$$

where $0<q<1$.

According to Lemma 1, for absolutely continuous function $a(t)$, the following equality can be obtained:

$$
\begin{aligned}
{ }_{0}^{C} D_{t}^{q}[a(t)]^{2}-2 a(t) & { }_{0}^{C} D_{t}^{q} a(t) \\
= & \frac{-q}{\Gamma(1-q)} \int_{0}^{t} \frac{d \tau}{(t-\tau)^{1-q}}\left[\int_{0}^{\tau} \frac{d a(\omega)}{(t-\omega)^{1-q}}\right]^{2} .
\end{aligned}
$$

Due to $\left(1 /(t-\tau)^{1-q}\right)\left[\int_{0}^{\tau}\left(d a(\omega) /(t-\omega)^{1-q}\right)\right]^{2} \geq 0$ for $\tau \in$ $[0, t]$, according to $(3)$, one has the following result:

$$
{ }_{0}^{C} D_{t}^{q}[a(t)]^{2}-2 a(t){ }_{0}^{C} D_{t}^{q} a(t) \leq 0 .
$$

Now, choosing the fractional-order BLDCM chaotic system (1) as drive system, we have the following main result. 
Theorem 2. Let the response fractional-order BLDCM system be

$$
\left(\begin{array}{c}
{ }_{0}^{C} D_{t}^{q} y_{d} \\
{ }_{0}^{C} D_{t}^{q} y_{q} \\
{ }_{0}^{C} D_{t}^{q} y_{a}
\end{array}\right)=\left(\begin{array}{c}
-\sigma y_{d}+y_{q} y_{a} \\
-y_{q}+\beta y_{a}-y_{d} y_{a} \\
\gamma\left(y_{q}-y_{a}\right)
\end{array}\right)+\Phi,
$$

where $0.96<q \leq 1, \Phi=\left(\begin{array}{c}-x_{q} \\ x_{d}+k-\beta-\gamma \\ 0\end{array}\right)\left(y_{a}-x_{a}\right)$ is a feedback controller, and $k$ is a real number. Choosing $k=2 k_{q} k_{a}, k_{q}, k_{a}$ are real numbers. If $\left|k_{q}\right|<1$ and $\left|k_{a}\right|<\sqrt{\gamma}$, then chaos synchronization between the response fractional-order BLDCM system (5) and the drive fractional-order BLDCM chaotic system (1) can be reached.

Proof. Let synchronization errors be $e_{d}(t)=y_{d}-x_{d}, e_{q}(t)=$ $y_{q}-x_{q}$, and $e_{a}(t)=y_{a}-x_{a}$. Thus, the error system between the response system (5) and the drive system (1) can be shown as follows:

$$
\begin{aligned}
& { }_{0}^{C} D_{t}^{q} e_{d}(t)=-\sigma e_{d}(t)+x_{a}(t) e_{q}(t), \\
& { }_{0}^{C} D_{t}^{q} e_{q}(t)=-e_{q}(t)-x_{a}(t) e_{d}(t)+(k-\gamma) e_{a}(t), \\
& { }_{0}^{C} D_{t}^{q} e_{a}(t)=\gamma\left[e_{q}(t)-e_{a}(t)\right] .
\end{aligned}
$$

Now, we choose one positive definite Lyapunov function as follows:

$$
L(t)=0.5\left[e_{d}^{2}(t)+e_{q}^{2}(t)+e_{a}^{2}(t)\right] .
$$
$L(t)$ is

Thus, the fractional derivative of the Lyapunov function

$$
{ }_{0}^{C} D_{t}^{q} L(t)=0.5\left[{ }_{0}^{C} D_{t}^{q} e_{d}^{2}(t)+{ }_{0}^{C} D_{t}^{q} e_{q}^{2}(t)+{ }_{0}^{C} D_{t}^{q} e_{a}^{2}(t)\right] .
$$

By inequality (4), according to (8), one has the following inequality:

$$
\begin{aligned}
{ }_{0}^{C} D_{t}^{q} L(t) \leq & e_{d}(t){ }_{0}^{C} D_{t}^{q} e_{d}(t)+e_{q}(t){ }_{0}^{C} D_{t}^{q} e_{q}(t) \\
& +e_{a}(t){ }_{0}^{C} D_{t}^{q} e_{a}(t) .
\end{aligned}
$$

By the error dynamical system (6), inequality (9) can be changed as follows:

$$
{ }_{0}^{C} D_{t}^{q} L(t) \leq-\sigma e_{d}^{2}(t)-e_{q}^{2}(t)-\gamma e_{a}^{2}(t)+k e_{q}(t) e_{a}(t) .
$$
follows:

Using $k=2 k_{q} k_{a}$, inequality (10) can be changed as

$$
\begin{aligned}
{ }_{0}^{C} D_{t}^{q} L(t) \leq & -\left[k_{q} e_{q}(t)+k_{a} e_{a}(t)\right]^{2}-\sigma e_{d}^{2}(t) \\
& -\left(1-k_{q}^{2}\right) e_{q}^{2}(t)-\left(\gamma-k_{a}^{2}\right) e_{a}^{2}(t) .
\end{aligned}
$$
that

Due to $\left|k_{q}\right|<1$ and $\left|k_{a}\right|<\sqrt{\gamma}$, thus, it can be obtained

$$
\begin{aligned}
& { }_{0}^{C} D_{t}^{q} L(t) \leq 0, \quad \forall e(t), \\
& { }_{0}^{C} D_{t}^{q} L(t)<0, \quad \forall e(t) \neq 0,
\end{aligned}
$$

where $e(t)=\left(e_{d}(t), e_{q}(t), e_{a}(t)\right)$.

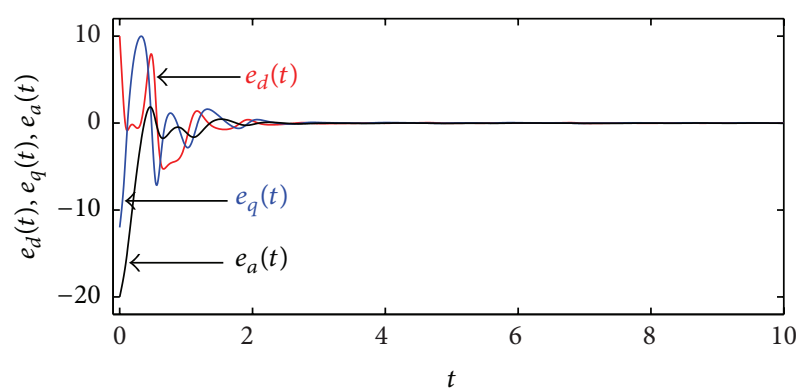

FIGURE 3: Evolution of the synchronization errors $e_{d}(t), e_{q}(t)$, and $e_{a}(t)$.

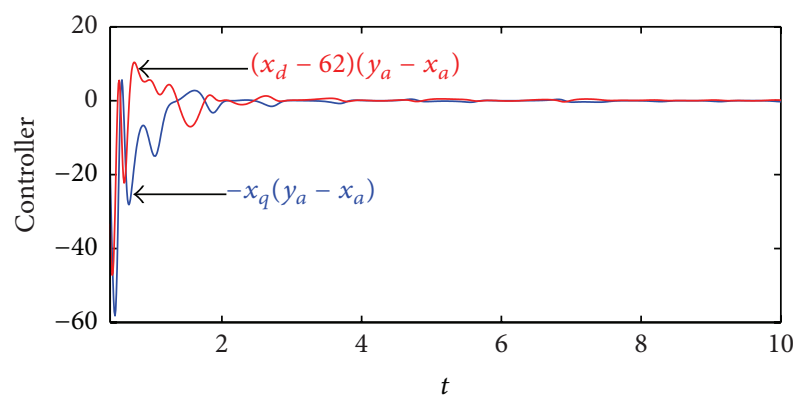

Figure 4: The time series for controller $\Phi$.

According to the stability of fractional order systems [13, 14], inequality (13) indicates that origin $\left(e_{d}(t), e_{q}(t), e_{a}(t)\right)=$ $(0,0,0)$ of error system (6) is asymptotically stable in the sense of Lyapunov. This result indicates that the chaos synchronization between the fractional-order BLDCM system (5) and the fractional-order BLDCM chaotic system (1) can be achieved. The proof is finished.

Remark 3. According to Theorem 2, if the chaos synchronization between the response system (5) and the drive system (1) is reached, the controller $\Phi=\left(\begin{array}{c}-x_{q} \\ x_{d}+k-\beta-\gamma \\ 0\end{array}\right)\left(y_{a}-x_{a}\right)$ could decrease to zero.

Next, simulation results are given. In numerical simulation, we set initial conditions as $\left(x_{d}, x_{q}, x_{a}\right)=(10,20,30)$ and $\left(y_{d}, y_{q}, y_{a}\right)=(20,8,10)$. The fractional-order $q$ is 0.975 in numerical simulation.

Case 1. Choosing $k_{q}=-0.8$ and $k_{a}=1.875$, thus $k=-3$ and $\Phi=\left(\begin{array}{c}-x_{q} \\ x_{d}-62 \\ 0\end{array}\right)\left(y_{a}-x_{a}\right)$. According to Theorem 2, the chaos synchronization between the response fractional-order BLDCM system (5) and the drive fractional-order BLDCM chaotic system (1) can be achieved. Figure 3 depicts the evolution of synchronization errors. Figure 4 shows the time series for controller $\Phi=\left(\begin{array}{c}-x_{q} \\ x_{d}-62 \\ 0\end{array}\right)\left(y_{a}-x_{a}\right)$.

Case 2. Choosing $k_{q}=k_{a}=0$, thus $k=0$ and $\Phi=$ $\left(\begin{array}{c}-x_{q} \\ x_{d}-59 \\ 0\end{array}\right)\left(y_{a}-x_{a}\right)$. According to Theorem 2, the response fractional-order BLDCM system (5) and the drive fractionalorder BLDCM chaotic system (1) can be synchronized. 


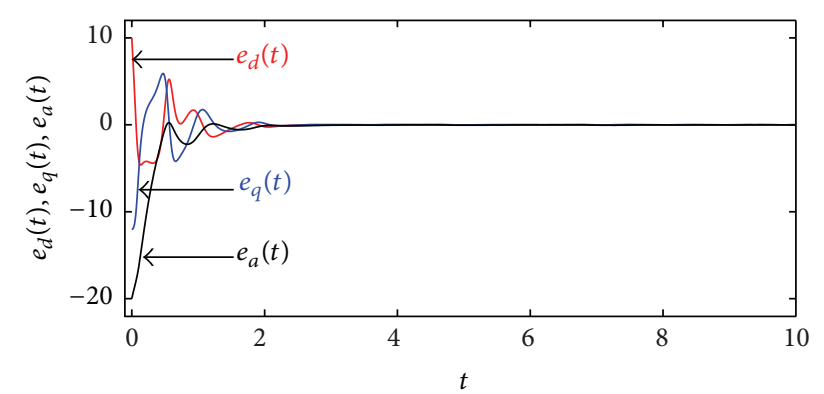

FIGURE 5: Evolution of the synchronization errors $e_{d}(t), e_{q}(t)$, and $e_{a}(t)$.

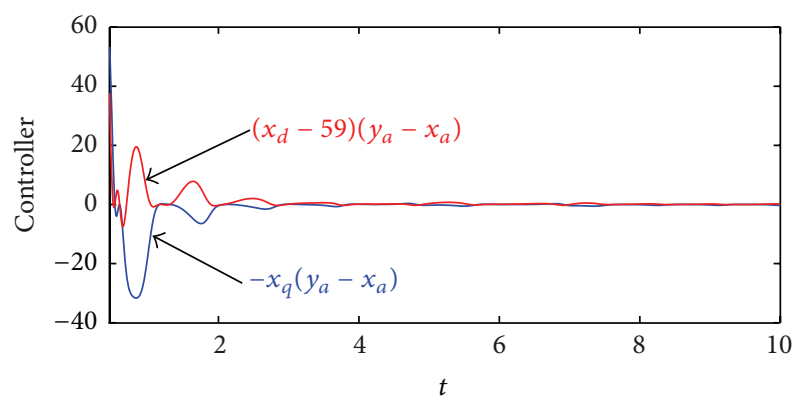

FIGURE 6: The time series for controller $\Phi$.

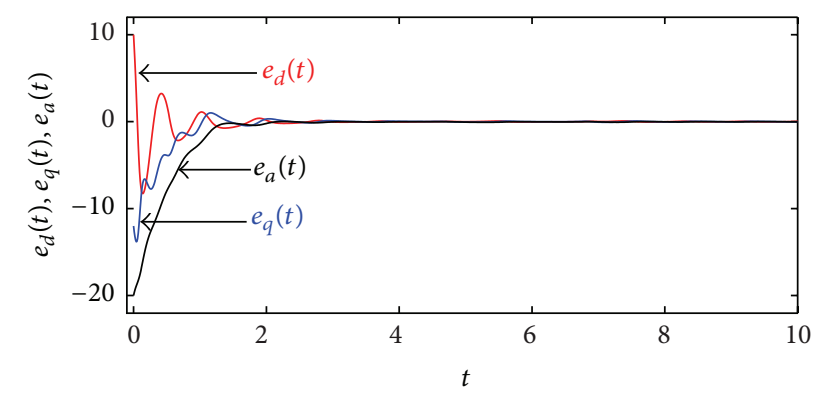

FIgURE 7: Evolution of the synchronization errors $e_{d}(t), e_{q}(t)$, and $e_{a}(t)$.

Figure 5 displays the evolution of synchronization errors. Figure 6 shows the time series for controller $\Phi=\left(\begin{array}{c}-x_{q} \\ x_{d}-59 \\ 0\end{array}\right)\left(y_{a}-\right.$ $\left.x_{a}\right)$.

Case 3. Choosing $k_{q}=0.9$ and $k_{a}=5 / 3$, thus $k=3$ and $\Phi=\left(\begin{array}{c}-x_{q} \\ x_{d}-56 \\ 0\end{array}\right)\left(y_{a}-x_{a}\right)$. According to Theorem 2, the chaos synchronization between the response fractional-order BLDCM system (5) and the drive fractional-order BLDCM chaotic system (1) can be arrived. Figure 7 displays the evolution of synchronization errors. Figure 8 shows the time series for controller $\Phi=\left(\begin{array}{c}-x_{q} \\ x_{d}-56 \\ 0\end{array}\right)\left(y_{a}-x_{a}\right)$.

According to Figures 3-8, the simulative results show the effectiveness of the proposed theorem in our paper.

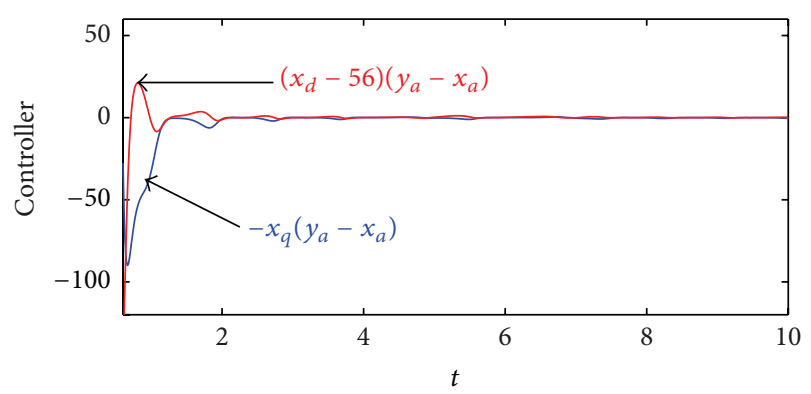

FIgURE 8: The time series for controller $\Phi$.

\section{Conclusions}

In this paper, the chaos synchronization for a fractional-order BLDCM chaotic system is discussed. One feedback controller is given. By the extension of Lyapunov direct method for nonlinear fractional-order system, a Lyapunov candidate function is established, and the theoretical proof of chaos synchronization is given. Finally, the numerical results are given, and it shows that the chaos synchronization scheme in our paper is effective. Up to now, to the best of our knowledge, there are no similar results on chaos synchronization of the fractional-order chaotic BLDCM system.

\section{Competing Interests}

The authors declare no competing interests.

\section{References}

[1] B. Hashtarkhani and M. P. Aghababa, "Introducing a new sliding manifold applied for control of uncertain nonlinear brushless DC and permanent magnet synchronous motors," Scientia Iranica, vol. 20, no. 6, pp. 2073-2083, 2013.

[2] Z.-M. Ge and C.-M. Chang, "Chaos synchronization and parameters identification of single time scale brushless DC motors," Chaos, Solitons and Fractals, vol. 20, no. 4, pp. 883-903, 2004.

[3] Z.-M. Ge and J.-W. Cheng, "Chaos synchronization and parameter identification of three time scales brushless DC motor system," Chaos, Solitons and Fractals, vol. 24, no. 2, pp. 597-616, 2005.

[4] Z.-M. Ge, C.-M. Chang, and Y.-S. Chen, "Anti-control of chaos of single time scale brushless dc motors and chaos synchronization of different order systems," Chaos, Solitons and Fractals, vol. 27, no. 5, pp. 1298-1315, 2006.

[5] J. Hu, L. Liu, and D.-W. Ma, "Robust nonlinear feedback control of a chaotic permanent-magnet synchronous motor with a load torque disturbance," Journal of the Korean Physical Society, vol. 65, no. 12, pp. 2132-2139, 2014.

[6] C.-L. Li, W. Li, and F.-D. Li, "Chaos induced in Brushless DC Motor via current time-delayed feedback," Optik, vol. 125, no. 21, pp. 6589-6593, 2014.

[7] S. H. Luo, S. L. Wu, and R. Z. Gao, "Chaos control of the brushless direct current motor using adaptive dynamic surface control based on neural network with the minimum weights," Chaos, vol. 25, no. 7, Article ID 073102, 2015. 
[8] M. Aziz, M. H. Tayarani-N, and M. Afsar, "A cycling chaosbased cryptic-free algorithm for image steganography," Nonlinear Dynamics, vol. 80, no. 3, pp. 1271-1290, 2015.

[9] M. Brindha and N. Ammasai Gounden, "A chaos based image encryption and lossless compression algorithm using hash table and Chinese Remainder Theorem," Applied Soft Computing Journal, vol. 40, pp. 379-390, 2016.

[10] A. Kiani-B, K. Fallahi, N. Pariz, and H. Leung, "A chaotic secure communication scheme using fractional chaotic systems based on an extended fractional Kalman filter," Communications in Nonlinear Science and Numerical Simulation, vol. 14, no. 3, pp. 863-879, 2009.

[11] P. Muthukumar, P. Balasubramaniam, and K. Ratnavelu, "Synchronization of a novel fractional order stretch-twist-fold (STF) flow chaotic system and its application to a new authenticated encryption scheme (AES)," Nonlinear Dynamics, vol. 77, pp. 1547-1559, 2014.

[12] P. Zhou, R.-J. Bai, and J.-M. Zheng, "Stabilization of a fractionalorder chaotic brushless DC motor via a single input," Nonlinear Dynamics, vol. 82, no. 1-2, pp. 519-525, 2015.

[13] N. Aguila-Camacho, M. A. Duarte-Mermoud, and J. A. Gallegos, "Lyapunov functions for fractional order systems," Communications in Nonlinear Science and Numerical Simulation, vol. 19, no. 9, pp. 2951-2957, 2014.

[14] Y. Li, Y. Chen, and I. Podlubny, "Stability of fractional-order nonlinear dynamic systems: lyapunov direct method and generalized Mittag-Leffler stability," Computers and Mathematics with Applications, vol. 59, no. 5, pp. 1810-1821, 2010.

[15] A. A. Alikhanov, "Boundary value problems for the diffusion equation of the variable order in differential and difference settings," Applied Mathematics and Computation, vol. 219, no. 8, pp. 3938-3946, 2012. 


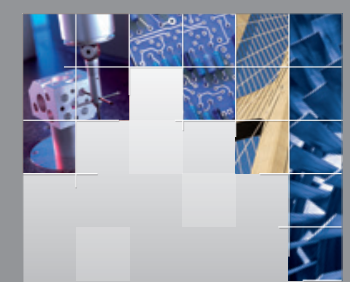

\section{Enfincering}
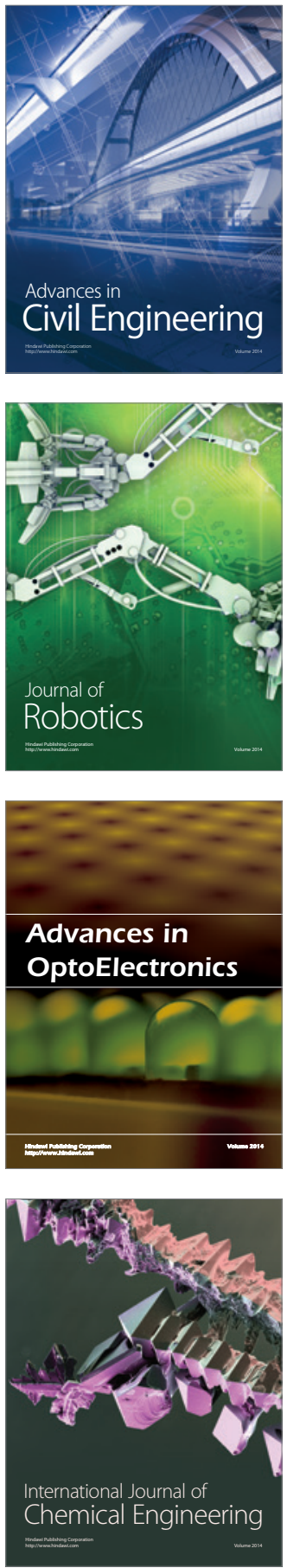

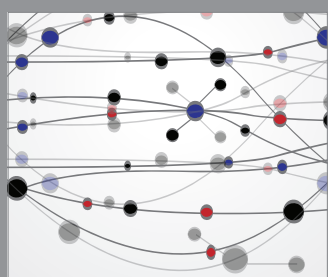

The Scientific World Journal

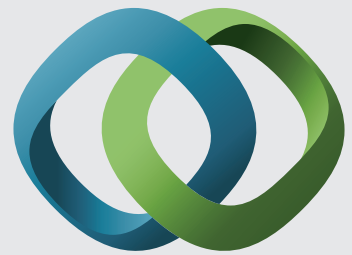

\section{Hindawi}

Submit your manuscripts at

http://www.hindawi.com
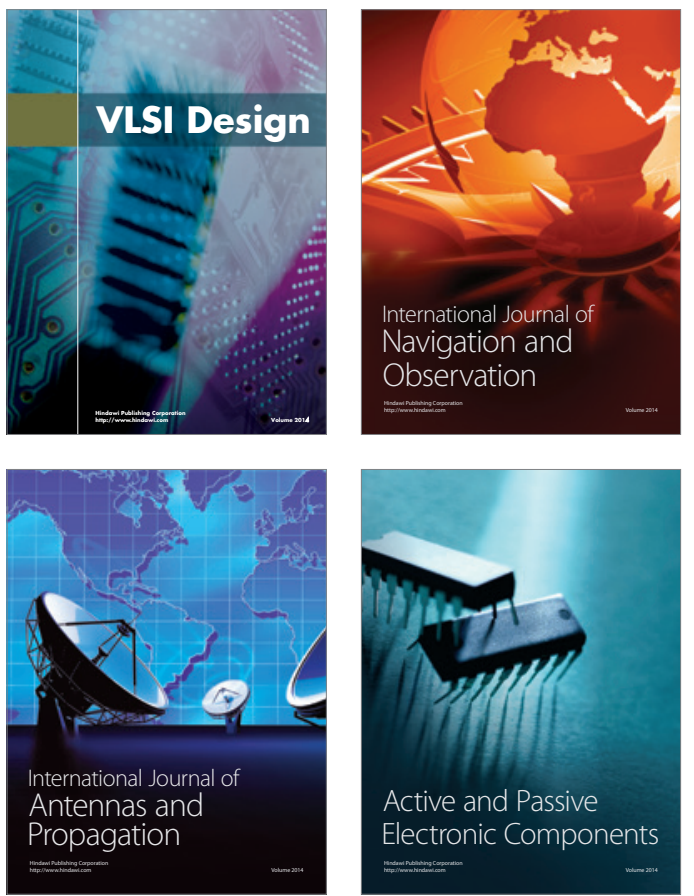
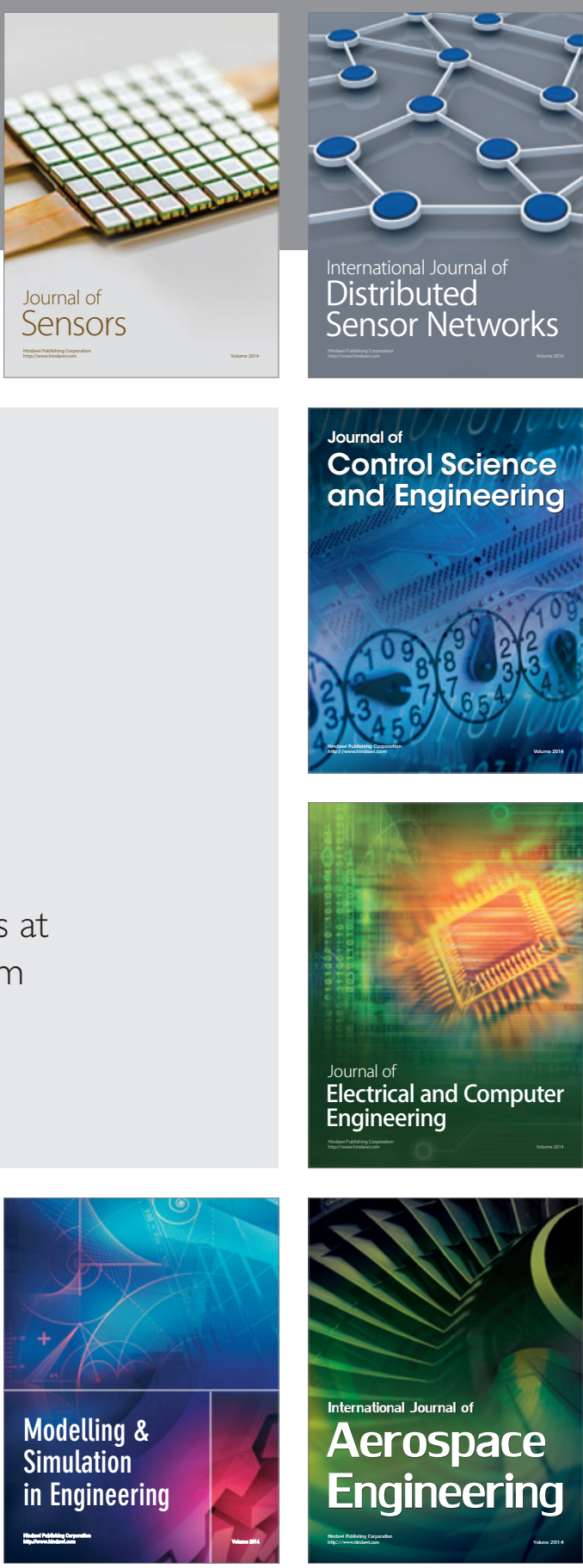

International Journal of

Distributed

Sensor Networks

Journal of

Control Science

and Engineering
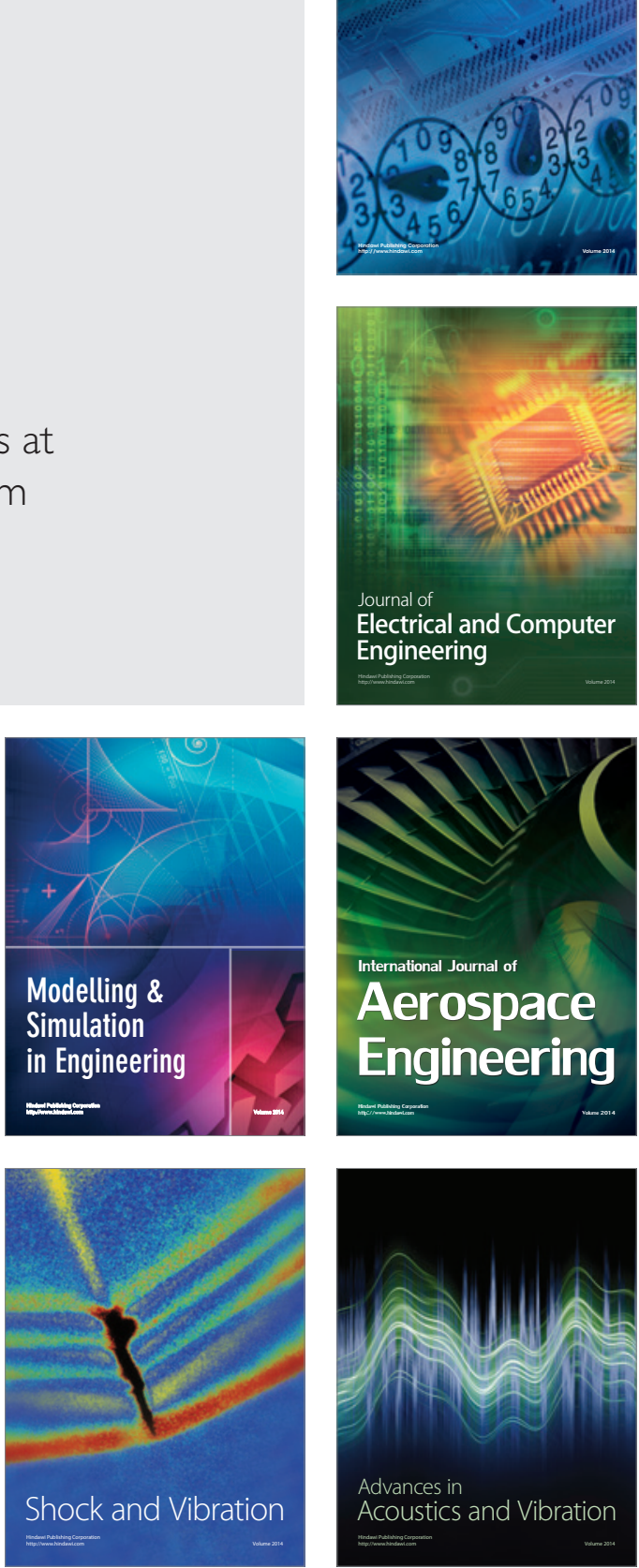